\title{
Electrophysiology of normal and pathological language processing
}

\author{
Sonja A. Kotz*, Angela D. Friederici \\ Max Planck Institute of Cognitive Neuroscience, P.O. Box 500 355, 04303 Leipzig, Germany
}

\begin{abstract}
The paper describes the three language-related event-related brain potentials (ERP) components: the N400 correlated with processes of semantic integration, the early mainly left accentuated anterior negativity correlated with initial structure building processes and the P600 reflecting processes of syntactic integration. These components are functionally specified with respect to normal comprehension. Moreover, the modulation of these components as well as their absence is discussed with respect to pathological language behavior. It is shown that the high temporal resolution of the ERP method allows not only to differentiate between early and late syntactic processes in normals, but also to specify the pathological changes of particular processes in patient groups.
\end{abstract}

(C) 2002 Elsevier Science Ltd. All rights reserved.

Keywords: Event-related brain potentials; Semantic; Syntactic; N400; P600

To comprehend language is one of the essential human capabilities. The process of language comprehension consists of a number of subprocesses covering different informational domains. Phonological information has to be processed before lexical access can take place. At the lexical level morphological and semantic information have to be taken into consideration (see Pulvermüller, 1999). Comprehension at the sentential level, moreover, requires the identification of the syntactic relations of different lexical elements in a sentence. The build up of these syntactic relations is based on two information types: first, the information about a word's syntactic category (i.e. noun, verb, determiner) whose identification is a necessary condition for local phrase structure building and second, morphosyntactic information such as inflectional morphology (i.e. number, person, gender, case) as well as verb argument information necessary to establish the relation between phrasal elements (noun phrases and verb). These different

\footnotetext{
* Corresponding author. Tel.: +49-3-41-99-40-231; fax: +49-3-41-99-40-113.

E-mail address: kotz@cns.mpg.de (S.A. Kotz).
} 
subprocesses during language comprehension have been correlated with distinct components in the event-related brain potential (ERP).

ERPs reflect the summation of the synchronous post-synaptic activity of large populations of neurons time-locked to critical stimulus events. To achieve a better signal-to-noise ratio for a given event, the brain's activity is averaged over a number of events of the same type. The time-locked average waveform typically displays a number of positive or negative peaks (polarity) of a particular latency post-stimulus onset and a characteristic scalp distribution. For example, the N400 component is a negative-going waveform with a latency of $400 \mathrm{~ms}$ after stimulus onset and a slightly right accentuated centro-parietal distribution at the scalp in visual semantic tasks and a more whole head distribution in acoustic semantic tasks. On the other hand the P600 is a positive-going waveform with a latency of $600 \mathrm{~ms}$ after stimulus onset with a bilateral centro-parietal distribution independent of modality in syntactic tasks. Thus, the polarity (negative or positive) as well as the latency and distribution of a component allow to dissociate cognitive processes associated with a particular component. The ERP in contrast to behavioral methods and other imaging methods such as PET and fMRI provide three dimensions as a dependent variable in normal populations: the latency measured in the milliseconds which reflects the timing of a particular cognitive process, the amplitude which indicates how easy a cognitive process can be integrated into a given context and the topography which allows to dissociate different cognitive processes that might result from one experimental manipulation or the differentiation of cognitive processes based on the activation of different neural structures. Changes within these dimensions may result from language pathologies and can be interpreted to reflect a slow down of a particular cognitive process (latency), a reduction in the efficiency of a particular process (amplitude) and a change in the cortical tissue supporting a particular process (topography). ERPs, in particular, allow to separate processes in time (i.e. milliseconds), which may be accumulated in other methods. Due to this separation, different subprocesses can be identified (i.e. early and late processes) and deficits can be functionally specified within these different subdomains. Of course, ultimately a combination of both ERPs or MEG with their high temporal resolution and fMRI or PET with their high spatial resolution would allow to draw a more comprehensive picture on the nature of cognitive processes in a specific network which can be complemented by ERP lesion data.

Within the language domain three different language-related ERP components have been identified: the so-called N400 shown to reflect lexical-semantic processes and the early mainly left accentuated anterior negativity (ELAN) and the late positivity (P600), both taken to reflect syntactic processes, representing early structure building and late integrational processes, respectively. In the following, we will first discuss the N400 and its related processing aspects before we turn to the two syntax-related components.

\section{The $\mathbf{N 4 0 0}$ as a marker of lexical-semantic processes}

Next to the processing of phonetic and syntactic information, the conveying of meaning is an important part of the comprehension process. With the help of ERPs a component has 
been identified that monitors lexical-semantic processes in lexical tasks (e.g. lexical decision) and/or lexical-semantic integration in semantic tasks: the N400 (e.g. Chwilla, Brown, \& Hagoort, 1995; Kutas \& Federmeier, 2000; Kutas \& Hillyard 1980, 1983). One specification of the N400 is that its amplitude varies as a function of context (word or sentence), meaning in the absence of context the N400 is larger than when context is present (see Kutas \& Van Petten, 1988, 1994 for reviews). This means that the more predictable a word becomes in a sentence context or at the word level (word frequency) the more the amplitude of the $\mathrm{N} 400$ will be reduced reflecting facilitated word processing. While the main N400 research has focused on normal language comprehension, the component has also been used as a tool to investigate lexical-semantic processing in aphasic and non-aphasic patients. Some of the studies with aphasic patients were partly motivated by the assumption that comprehension problems in Broca's aphasics and Wernicke's aphasics are related to changes or impairments in the time course of lexical integration (e.g. Hagoort, 1993), and partly by the hypothesis the patients with temporal lobe lesions suffer from a lexical-semantic deficit in particular (e.g. Friederici, Hahne, \& von Cramon, 1998; Revonsuo \& Laine, 1996). In addition, studies with non-aphasic language patients (e.g. Kotz, Friederici, \& von Cramon, 1999) attempted a localization of the N400 generator via lesion studies guided by evidence from intracranial recordings that imply the anterior temporal lobe as a neural source of the N400 (e.g. Nobre \& McCarthy, 1995 ) and the hypothesis that the knowledge representation of nouns is correlated with activation in the left anterior and posterior temporal lobe (Damasio, Grabowski, Tranel, Hichwa, \& Damasio, 1996).

Before we report patient data on the N400 it is important to raise a note of caution as to how modulations of the N400 can be interpreted in patients. The application of ERPs in patient studies aims at characterizing cognitive deficits on a neurological basis. However, the lack of an ERP effect in patients does not necessarily mean that no activation of

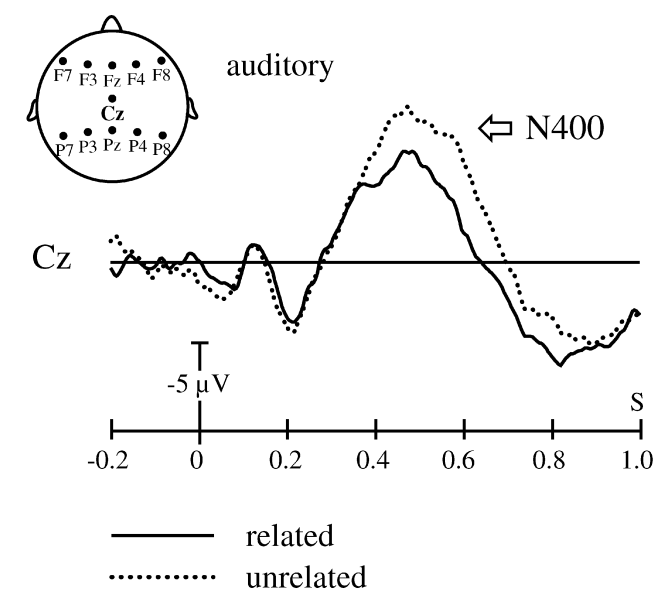

Fig. 1. Displays the auditory N400 component elicited in a semantic priming paradigm at a selected electrode-site $(\mathrm{Cz})$. The vertical line indicates the onset of a related target word (straight line) as compared to an unrelated target word (dotted line), while the horizontal line shows the time course from $200 \mathrm{~ms}$ prestimulus onset to $1000 \mathrm{~ms}$ after stimulus onset. Adapted from Kotz et al. (1999). 
cognitive and neural systems has taken place. Rather, the multitude of parameters that define a component, such as the amplitude, the latency and the distribution are helpful to specify the relationship between a component and the underlying cognitive and neural mechanisms. Thus, either a clear structural or a clear functional deficit allow to investigate the relationship between a particular ERP component and a cognitive process. Thus, we present data that used either structural and functional deficits as a selection criterion for N400 language studies in patients.

Studies based on structural deficits. In two single case studies, ERPs were recorded from patients with temporo-parietal lesions. Revonsuo \& Laine (1996) reported that the N400 effect was reduced in an auditory semantic priming experiment and concluded that this is evidence for a lexical-semantic deficit resulting from left temporal lesions. On the other hand, Friederici et al. (1998) reported that a patient with the temporo-parietal lesion displayed a very late positivity $(1000-1500 \mathrm{~ms})$ rather than an N400 indicating that the patient reacted to the semantic incompatibility of the target word in the sentence (e.g. The jam was murdered), while a patient with a left anterior lesion showed an N400 that was similar to normal controls. The modulation of the N400 effect could be connected to the finding in an off-line experiment presenting the same sentences in which the patient with the temporal lesion reported a 'feeling of knowing' that there were errors in the sentences, but that she was not able to indicate which element caused an error.

An N400 comparable to normal controls was also reported for two groups of patients with left anterior lesions, e.g. for a group in which lesions were restricted to subcortical structures and for another group which included patients with cortical lesions involving Broca's area and adjacent tissue in addition to subcortical structures (Friederici, von Cramon, \& Kotz, 1999). These findings suggest that left anterior cortical regions are not necessarily involved in processes reflected by the N400 and that, in turn, patients with lesions in the anterior part of the left hemisphere do not primarily suffer from an impairment of lexical-semantic integration processes.

Based on data from intracranial recordings (e.g. Nobre \& McCarthy, 1995) which suggest an involvement of the anterior temporal lobe in the generation of the N400, Kotz et al. (1999) compared non-aphasic language patients with left and right anterior temporal lobe lesions and normal age-matched controls in a word list priming experiment by manipulating semantic information types (associative functional-chain-lock, associative categorical-cat-dog, categorical-horse-dog). Words were presented auditorily with a stimulus onset asynchrony (SOA) of $1025 \mathrm{~ms}$ and subjects were required to press a button when they heard a verb or an adjective (10\%).

Normal controls made fewer errors detecting targets in the word list. They showed the expected associative (functional/categorical) and purely categorical N400 priming effects. Overall, the error rate between left and right anterior temporal lesions patients was comparable, but higher than in normal controls. Patients with left anterior temporal lesions displayed a very small associative functional priming effect, an associative categorical priming effect (extended latency) and a delayed categorical priming effect. Patients with right anterior temporal lobe lesions, in contrast, showed an associative functional priming effect, a smaller associative categorical priming effect, but no categorical priming effect. In particular, the N400 priming effects that involved categorical information were modulated by a positivity around $200 \mathrm{~ms}$ at left hemisphere electrode-sites (Fig. 1) in the 


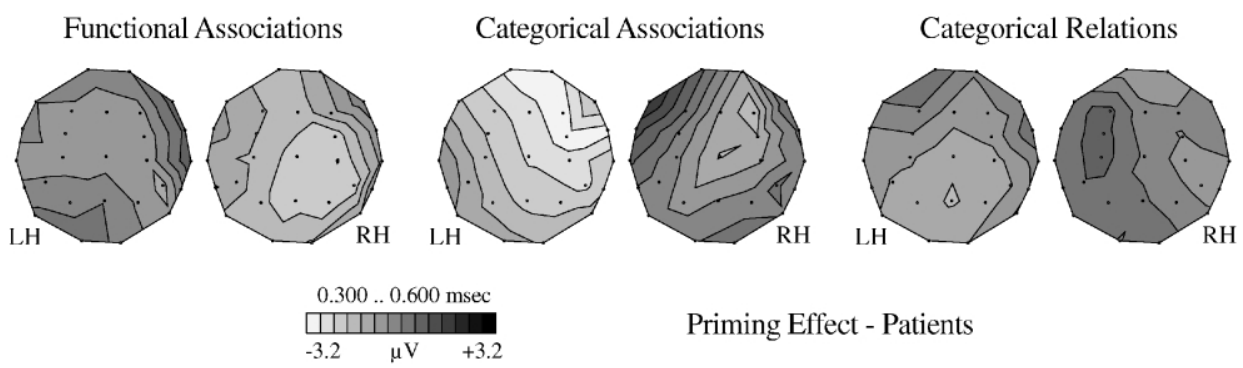

Fig. 2. Displayed are ERP difference maps (300-600 ms) for the left anterior temporal (LH) and the right anterior temporal (RH) patient group comparing functional associative, categorical associative and categorical N400 priming effects in a word list priming task. The effect size is indicated in $\mu \mathrm{V}$ as a negative polarity (lighter shades of gray) and positive polarity (darker shades of gray). Adapted from Kotz et al. (1999).

right anterior temporal group. These data indicate that word list priming in non-aphasic patients with anterior temporal lobe lesions varies as a function of semantic information type. The different ERP (Fig. 2) patterns for the two patient groups suggest that the left anterior temporal lobe might monitor associative information processing, while the right anterior temporal lobe might be involved in categorical information processing.

In summary, it appears that anterior and posterior parts of the temporal lobe are engaged in the processing of lexical-semantic information as reflected in the N400 component. Furthermore, it is apparent that the modulation of the N400 varied mainly as a function of amplitude and latency, but also in interaction with earlier components. This might imply that other cognitive processes come into play in order to process lexicalsemantic information when a patient suffers from a temporal lobe lesion.

Studies based on functional deficits. In contrast to some of the earlier described findings is some recent data reported by Hagoort and colleagues (Hagoort, Brown, \& Swaab, 1996; Swaab, Brown, \& Hagoort, 1997, 1998). Hagoort et al. (1996) used an auditory word pair priming paradigm to investigate lexical-semantic integration processes in aphasic patients. Three patient groups and matched normal controls were tested: (1) patients with left hemisphere lesions diagnosed as Broca's aphasics, (2) patients with left hemisphere lesions diagnosed as Wernicke's aphasics, and (3) patients with right hemisphere lesions. The prime-target pairs presented were either associatively related (i.e. bread-butter) or semantically related (e.g. church-villa). For these word pairs elderly normal controls displayed an N400 priming effect for both prime-target types. The patients ERP pattern was compared to this N400 effect in a number of ways. In a first analysis, left hemisphere patients were grouped according to their clinical diagnosis based on the Aachen Aphasic Test. In this analysis a statistically reliable N400 was found in the Broca patient group for associatively and for semantically related targets. In the Wernicke patient group the N400 was also present, but clearly reduced compared to normal controls. However, as no significant between-subjects interaction was found, aphasic patients were regrouped in a second analysis. This second analysis compared patients with high comprehension and patients with low comprehension (based on the comprehension score of the Aachen Aphasic Test), independent of the clinical diagnosis or the lesion site. Again, both groups showed a significant N400 priming effect for both word pair types, but the effect was larger 
for the high comprehenders than for the low comprehenders which was confirmed in significant interaction with the factor group. Patients with right hemisphere lesions displayed a differential N400 priming effect, namely only for associative targets. The authors concluded that patients with clear comprehension deficits (e.g. low comprehenders) show a reduction of the $\mathrm{N} 400$ effect 'indicating a reduction in their ability to match words for their semantic similarity' (p. 643). As the mechanism underlying semantic matching in a word priming paradigm is similar to integration processes involved in sentence processing, these patients' deficits may arise from an impairment of integrating word meanings into the current overall representation of sentences.

Swaab et al. (1997, 1998) further investigated lexical-integration processes in Broca's aphasics at the sentence level. With these two studies they aimed to contribute to the ongoing discussion about the nature of the processing deficit underlying Broca's aphasics' comprehension difficulties. While some researchers propose that the underlying deficit could be due to a delayed access of syntactic information in particular (e.g. Friederici, 1985; Friederici \& Kilborn, 1989; Haarmann \& Kolk, 1991, 1994) others have claimed that Broca's aphasics' comprehension deficit may be due either to a delayed access to the semantic lexicon caused by a loss of automatic retrieval mechanisms (Milberg, Blumstein, \& Dworetzky, 1987; Milberg, Blumstein, Katz, Gershberg, \& Brown, 1995), to a nonexhaustive access of words in the lexicon (Swinney, Zurif, \& Nicol, 1989), or to an impaired lexical integration processes (Hagoort, 1993; Tyler, Ostrin, Cooke, \& Moss, 1995). In one study Swaab et al. (1997) auditorily presented sentences which were either correct or contained a semantic anomaly (i.e. The girl dropped the candy on the sky). The study included Broca's aphasics, Wernicke's aphasics, right hemisphere patients, and normal controls. Aphasic patients were grouped into high and low comprehenders regardless of the clinical syndrome or structural deficit. The results showed that agematched, non-aphasic right hemisphere patients, aphasic patients with high comprehension showed essentially the same N400 effect. Aphasic patients with low comprehension also displayed an N400 effect, though this effect was delayed by about $100 \mathrm{~ms}$. It was concluded that the comprehension deficit for the low comprehenders is caused by a delay in lexical integration.

In a further study Swaab et al. (1998) investigated whether Broca's aphasics comprehension problem is due to an inability to exhaustively access word meaning or due to a slower-than-normal rise time for lexical processes. Based on behavioral data from a cross-model lexical decision paradigm Swinney et al. (1989) had proposed these two alternatives as a possible explanation for the comprehension impairment in Broca's aphasics. Swaab et al. (1998) presented ambiguous words (e.g. bank) in three different context conditions followed by a target word (e.g. river): these are sentence contexts raising the meaning of the sentence-final ambiguous word that was (a) related to the target (concordant condition), (b) incompatible with the target (discordant condition), or (c) a sentence context containing a non-ambiguous word which was unrelated to the target. Examples of these different conditions are: (a) concordant: The man planted a tree on the bank, (b) discordant: The man made a phone call to the bank, and (c) The boy petted the dog on its head. Subjects were required to attentively listen to the auditory input, no further task was applied. The activational status of the ambiguous word was inferred from the amplitude of the N400 to the target word. The interstimulus interval (ISI) between the 
sentence final word and the target word varied, either by 100 or $1200 \mathrm{~ms}$. At the short ISI, the amplitude of the $\mathrm{N} 400$ was reduced in the concordant condition relative to the unrelated and the disconcordant conditions in normals, whereas no significant difference was observed for the latter two conditions. Broca's aphasics also displayed a reduced N400 amplitude for the concordant as compared to the unrelated and the discordant condition. In contrast to the normal controls that showed no significant reduction of the N400 amplitude in the discordant condition relative to the unrelated condition, Broca's aphasics showed a substantial difference between these two conditions. Separate analyses for high and low comprehenders of the aphasic patients did not reveal a significant interaction with the factor group. At the long ISI, the amplitude of the N400 to the target in the concordant condition was reduced relative to the unrelated condition. The N400 to targets in the discordant condition started to diverge after $550 \mathrm{~ms}$ post-stimulus onset. Just as normals, Broca's aphasics showed a reduction of the N400 amplitude for targets in the concordant relative to the unrelated and discordant conditions. No difference was found for the latter two conditions. Additional analyses introducing the factor of high versus low comprehenders did not show any interaction with this factor. The differential finding for Broca patients in the short and the long ISI with respect to the discordant versus the unrelated condition was taken to show that at a short ISI Broca's aphasics did not successfully select the appropriate meaning of the ambiguous word (indicated by the difference between the two conditions) although they did at a long ISI (indicated by the non-difference between the two conditions). From these data the authors concluded that Broca's aphasics are delayed in the process of contextual selection. Although this finding is also compatible with a view of an unsuccessful inhibition of the inappropriate meaning, they take this finding to support the idea that comprehension deficits in Broca's aphasic result from a delay in the process of integrating lexical information.

\section{The ELAN and P600 as markers of syntactic processes}

Central to the process of language comprehension is the correct identification of syntactic information. This was shown quite impressively by an early study on aphasic language comprehension. Caramazza and Zurif (1976) tested agrammatic Broca patients with sentences which cannot be interpreted correctly without the identification of the underlying syntactic structure (e.g. The girl the boy is chasing is tall). Knowledge about the structure is necessary to understand such a sentence as a simple agent-first strategy will lead to the wrong interpretation. Broca patients systematically demonstrated an incorrect interpretation of such sentences. Different explanations have been given as the underlying cause for this behavioral deficit. While some researchers assume that it is due to a slowed lexical access to syntactically relevant information (Friederici \& Kilborn, 1989; Haarmann \& Kolk, 1991, 1994, see also Swinney et al., 1989) others postulate that it is due to a problem establishing long distance dependencies necessary to process noncanonical sentence (Grodzinsky, 2000). While the first view would predict problems in online local phrase structure building, the second view predicts problems in processes of structural integration. Thus, it appears that processes of structure building (e.g. phrase structure) and structure integration or repair are necessary for adequate comprehension. 
There are two ERP components that have been identified to vary as a function of syntactic processes. (1) An ELAN either present between 100 and $200 \mathrm{~ms}$ or between 300 and $500 \mathrm{~ms}$, and (2), a late positivity with a peak latency around $600 \mathrm{~ms}$ or later. Late positivities have been found for the processing of infrequent sentence structures (Hagoort, Brown, \& Groothusen, 1993; Mecklinger, Schriefers, Steinhauer, \& Friederici, 1995; Osterhout \& Holcomb, 1992, 1993; Osterhout, Holcomb, \& Swinney, 1994) as well as for the processing of syntactically incorrect structures (Friederici, Pfeifer, \& Hahne, 1993; Neville, Nicol, Barss, Forster, \& Garrett, 1991; Osterhout \& Mobley, 1995). In the following the functional relevance of the left anterior negativity and the late positivity will be specified on the basis of ERP results from normals before we turn to the ERP pattern of aphasic language pathologies.

(1) An early study by Neville et al. (1991) investigated the processing of syntactic violations in a number of different sentence structures in a sentence reading ERP experiment. This study used a rapid serial visual presentation mode with a presentation time of $300 \mathrm{~ms}$ and an ISI of only $200 \mathrm{~ms}$. The violation of phrase structure, in particular, elicited a left anterior negativity (around $125 \mathrm{~ms}$ ) which was followed by a left temporoparietal negativity between 350 and $500 \mathrm{~ms}$. This early left anterior negativity was evoked by a word category error (i.e. Max's of proof the theorem). As the language input is processed from left to right, the parser detects a word category error when perceiving 'of' as the rules of English do not license this function word to follow a genitive 's'.

A more recent visual study that investigated phrase structure violations in German with a fast presentation mode found an early left anterior negativity between 100 and $200 \mathrm{~ms}$, however, only when stimulus items were presented with high visual contrast (Gunter, Friederici, \& Hahne, 1999). When presented with low visual contrast a left anterior negativity between 300 and $500 \mathrm{~ms}$ was elicited. Left anterior negativities between 300 and $700 \mathrm{~ms}$ have been reported in other studies using a relative slow visual presentation mode. For example, Münte, Heinze, \& Mangun (1993) also investigated word category violations (i.e. your write) and reported a left anterior negativity after $300 \mathrm{~ms}$. The combined data suggest that the early anterior negativity which seems to reflect early syntactic processes may only be observable in the visual word-by-word presentation mode when the input is fast and of optimal visual quality. Only with this input condition may early syntactic processes that are assumed to be fast and automatic be triggered.

An early mainly left anterior negativity between 100 and $200 \mathrm{~ms}$ has been repeatedly reported for phrase structure violations in the auditory domain when connected speech is presented (Fig. 3). Friederici and colleagues (Friederici et al., 1993; Hahne \& Friederici, 1999) presented syntactically correct and incorrect sentences as connected speech. Syntactic incorrectness was realized as a word category violation (i.e. Der Freund wurde im besucht/The friend was in the visited). The ELAN was interpreted to reflect highly automatic processes of initial structure building, i.e. so-called first pass parsing processes (Friederici, 1995). Support for this proposal comes from a study which found the ELAN to be unaffected by attentional factors (Hahne \& Friederici, 1999). The study varied the proportion of the number of correct and incorrect sentences in two experimental conditions (20 and $80 \%$ incorrect). The ELAN was present in both conditions, equal in amplitude and in latency, indicating that those processes that are reflected by the early negativity are highly automatic. 


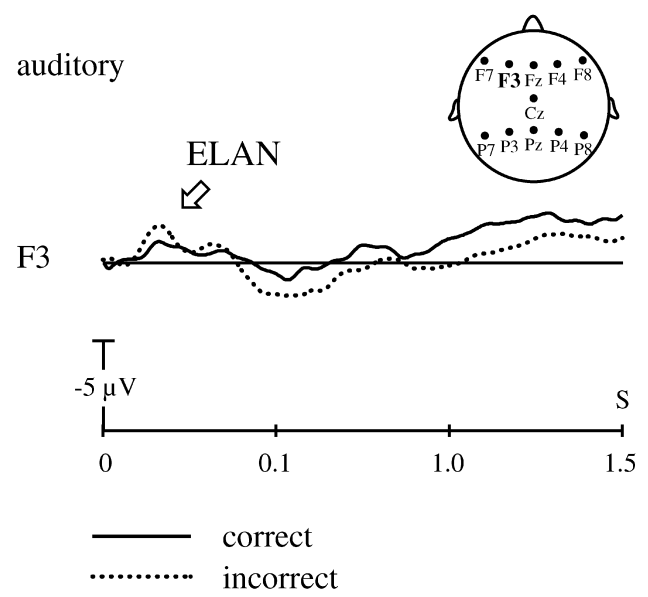

Fig. 3. Displays the auditory ELAN component elicited in a sentence judgment task at a selected electrode-site (F3). The vertical line indicates the onset of the correct target word (straight line) as compared to the incorrect target word (dotted line), while the horizontal line shows the time line from stimulus onset to 1500 post-stimulus onset. Adapted from Friederici et al. (1999).

The literature reviewed so far may suggest that negativities elicited by phrase structure violations are always of short onset latency as long as the input is fast. ${ }^{1}$

A number of different syntactic aspects have been studied in a variety of ERP experiments, all using a word-by-word visual presentation mode. The violation types investigated in these studies were agreement violations and argument-structure violations. Agreement violations were investigated in English (Kutas \& Hillyard, 1983; Osterhout \& Mobley, 1995) in Dutch (Gunter, Stowe, \& Mulder, 1997; Hagoort et al., 1993) and in German (Penke et al., 1997). With the exception of one study (Hagoort et al., 1993) all reported a negativity between 300 and $500 \mathrm{~ms}$ which was followed by a late positivity. Most of these negativities displayed a fronto-central or frontal maximum, often with a left dominance. This left anterior negativity has been labeled LAN (Coulson, King, \& Kutas, 1998). A similar pattern was also found for inflectional errors during auditory sentence presentation (Friederici et al., 1993). The combined data seem to suggest that morphosyntactic violations realized an as incongruency of the inflection elicit a left anterior negativity around $400 \mathrm{~ms}$ followed by a late positivity independent of the input modality.

\footnotetext{
${ }^{1}$ Note that the detection of this violation requires the identification of the critical word's syntactic word category (e.g. verb versus noun). Languages, however, differ with respect to when during word perception this information becomes available. This information could be coded in the prefix (e.g. gefischt/fished versus Fisch/fish) in the word stem (e.g. eat versus meal), or in the suffix (e.g. refined versus refinement). During auditory input the word is processed left-to-right. Thus the latency of the early anterior negativity will not be independent from when information about the word category is available, early or late in the word. This was shown in a study by Friederici, Hahne, and Mecklinger (1996) in which the phrase structure violation dependent left anterior negativity was realized by words in which the word category information was marked in the suffix (refined versus refinement). The left anterior negativity was late when measured from the word onset, but early when measured from the word category identification point.
} 


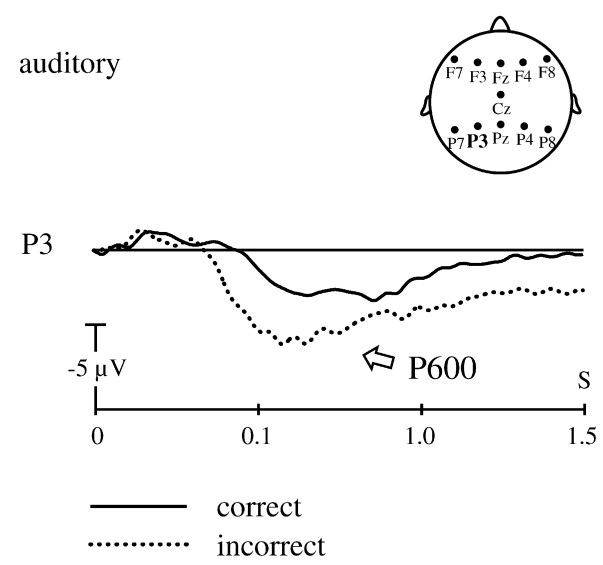

Fig. 4. Displays the auditory P600 component elicited in a sentence judgment task at a selected electrode-site (P3). The vertical line indicates the onset of the correct target word (straight line) as compared to the incorrect target word (dotted line), while the horizontal line shows the time line from stimulus onset to 1500 post-stimulus onset. Adapted from Friederici et al. (1999).

Violations of the verb-argument-structure are also correlated with a left anterior negativity around $400 \mathrm{~ms}$ (Rösler, Friederici, Pütz, \& Hahne, 1993). Rösler et al. (1993) interpreted the left anterior negativity as a correlate for processes associated with the information about a verb's argument structure. A similar left anterior negativity followed by a late positivity was reported by Coulson et al. (1998) for incorrect case-marked elements causing a mismatch between the verb's argument structure and its argument. The combined data suggest that left anterior negativities correlate systematically with syntactic aspects, namely phrase structure violations and morphosyntactic violations. Everything else being equal (e.g. presentation mode, etc.) the latency of the early anterior negativity appears to be dependent on the violation type with phrase structure violations evoking an early mainly ELAN and morphosyntactic violations evoking a later left anterior negativity (LAN).

(2) Late positivities have been observed with outright syntactic violations and with violations of structural preferences. The latter violations can be realized in temporarily ambiguous structures, which are disambiguated at some point in the sentences towards the non-preferred reading. Osterhout and Holcomb (1992) reported a late positivity for socalled garden-path sentences, such as (i.e. The broker persuaded to sell the stock). At the disambiguating element to it is clear that the underlying structure of the sentence is not a simple subject-verb-object structure (i.e. The broker persuaded the man). This element evoked a centro-parietal positivity around $600 \mathrm{~ms}$ (see Fig. 4). It was labeled the P600 component. From these and other studies (Osterhout \& Holcomb, 1993; Osterhout et al., 1994) Osterhout and colleagues developed the view that the late positivity (P600) is a marker of the garden-path effect and that this effect is found whenever the parser has to revise a structure.

This interpretation receives support from a number of studies which investigated the processing of complex non-preferred, but correct sentence structures in German (e.g. Mecklinger et al., 1995). Thus, from these studies it appears that the P600 is present in the 
absence of a left anterior negativity when sentences are correct, but requires a revision of the underlying structure at a given point in time. In addition, the P600 is observed following a left anterior negativity in the presence of an outright violation (Coulson et al., 1998; Friederici et al., 1993; Neville et al., 1991; Osterhout \& Mobley, 1995; Rösler et al., 1993).

In summary, it appears that left anterior negativities are specific for on-line syntactic processes whereas the $\mathrm{P} 600$ reflects processes of syntactic integration (for a similar argument see Coulson et al., 1998; Friederici, 1995; Kaan, Harris, Gibson, \& Holcomb, 2000; Münte, Heinze, Matzke, Wieringa, \& Johannes, 1998). The left anterior negativity is absent when there is no syntactic violation, it varies in its latency as a function of the availability of the syntactic information, but remains unaffected by extra-linguistic variables such as probability and task demands. The P600 present for syntactic violations and violations of syntactic preferences, in contrast, varies as a function of probability and task demands.

Studies based on structural deficits. Although the studies investigating syntactic processes in aphasic patients using ERPs are limited, they prove the potential of ERPs for the investigation of impairments of different language-related subprocesses such as the early automatic parsing process and the late process of syntactic integration. One of the hypotheses for the explanation of agrammatic comprehension was that Broca patients have lost the ability to access syntactic information during on-line processing. This hypothesis was tested in a study in which syntactically incorrect (phrase structure violation) and semantically incorrect (selectional restriction violation) and correct sentences where presented to two aphasic cases: a Broca patient with an extended lesion restricted to the anterior part of the left hemisphere, and a Wernicke patient with a circumscribed lesion in the posterior part of the left hemisphere (Friederici et al., 1998). Sentences were presented auditorily as connected speech and subjects were required to indicate whether a sentence was correct or not. Normal controls showed an ELAN followed by a P600 in the syntactic violation condition, and an N400 in the semantic violation condition. The ERP pattern for the two patients differed from normals and displayed a double dissociation when compared to each other. The Wernicke patient showed an ELAN followed by a (very) late positivity in the syntactic condition, but no N400 in the semantic condition. In contrast, for the Broca patient no ELAN was found, but a P600 and an N400. From these data it was concluded that left temporo-parietal areas support lexical-semantic integration processes and that the comprehension problem of the Wernicke patient was due to a failure of the lexical-semantic integration processes. The Broca patient's deficit was defined as a loss of the fast and automatic initial structure building processes indicated by the absence of the ELAN. The presence of the P600 in this patient suggests that secondary syntactic processes are not lost, a view which was backed up by the finding of a relatively good performance on the grammaticality judgment task.

In a further study two groups of patients with left anterior lesions and residual aphasia as well as normal controls were tested with sentences containing syntactic, semantic or no violation. The two patient groups differed with respect to their lesion sites: one group had left anterior lesions partly including the basal ganglia (cortical group) whereas the other group had left subcortical lesions restricted to the basal ganglia (basal ganglia group) 
Cortical Group
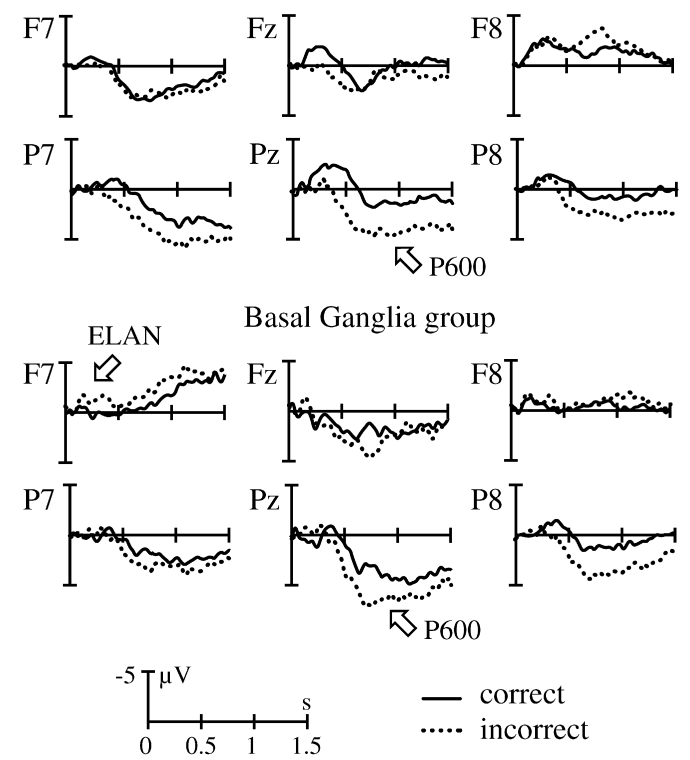

Fig. 5. Displayed are the average waveforms for the ELAN and the P600 components at selected left and right fronto-parietal electrode-sites for the cortical group (top) and the basal ganglia group (bottom) for the syntactic condition. The waveforms are superimposed for the correct (straight line) and the incorrect (dotted line) condition. The vertical lines indicate the onset of the critical word. The first $100 \mathrm{~ms}$ post-stimulus onset are used as a baseline. Adapted from Friederici et al. (1999).

(Friederici et al., 1999). This study investigated the involvement of the left basal ganglia in the neuronal network supporting on-line language comprehension.

The results were straight forward (Fig. 5): normal age-matched controls displayed the expected pattern of an early anterior negativity (though less clearly lateralized than in other studies) followed by a P600 in the syntactic condition and an N400 in the semantic condition. The ERP pattern for the basal ganglia group was similar to that of the normal controls: there was an early anterior negativity followed by a P600 in the syntactic violation condition that was more reduced in amplitude than in the normals and an N400 in the semantic violation condition. Patients with left frontal cortical lesions displayed an attenuated N400, a P600, but no early anterior negativity. Under the assumption that the early anterior negativity reflects automatic first-pass parsing processes and the P600 component more controlled second-pass parsing processes, these results suggest that the left frontal cortex might support early parsing processes, and that specific regions of the basal ganglia, in contrast, may not be crucial for early parsing processes during sentence comprehension.

An additional study investigated the possible involvement of the basal ganglia in language comprehension examined patients with no focal lesions but with Parkinson's disease (PD) (Friederici, Kotz, Werheid, Hein, \& von Cramon, under revision). The study used sentence material similar to the previous one. Both normals and PD patients showed an ELAN and an N400. The P600, however, was modulated in the PD patients. These data 
suggest that the deficit underlying pathological language behavior in PD patients (e.g. Grossman et al., 1991; Grossman, Carvell, Stern, Gollomp, \& Hurtig, 1992; Ullman et al., 1997) may not be caused by impaired automatic structure building processes, but rather by deficiencies in late processes of syntactic integration. This view is compatible with the notion advanced by Grossman et al. (1992) that the basic problem underlying PD comprehension lies in deficient attentional mechanisms whereas preattentional processes are intact.

\section{Conclusion}

The summarized data presented here indicate that electrophysiological measures allow to identify semantic as well as early and late syntactic processes during language comprehension via specific ERP components. The strength of this tool lies in the temporal resolution which not only provides the possibility to differentiate early from late syntactic processes, but also allows to specify the pathological delay of a particular process in the millisecond domain.

\section{Acknowledgments}

This study was supported by the Leibniz Science Prize awarded to A.F. by the Deutsche Forschungsgemeinschaft (German Research Foundation, DFG).

\section{References}

Caramazza, A., \& Zurif, E. B. (1976). Dissociation of algorithmic and heuristic processes in language comprehension: Evidence from aphasia. Brain and Language, 3, 572-582.

Chwilla, D. J., Brown, C., \& Hagoort, P. (1995). The N400 as a function of the level of processing. Psychophysiology, 32, 274-285.

Coulson, S., King, J., \& Kutas, M. (1998). Expect the unexpected: Eventrelated brain responses of morphosyntactic violations. Language and Cognitive Proesses, 13, 21-58.

Damasio, H., Grabowski, T. J., Tranel, D., Hichwa, R. D., \& Damasio, A. R. (1996). A neural basis for lexical retrieval. Nature, 380, 499-505.

Friederici, A. D. (1985). Levels of processing and vocabulary types: Evidence from on-line comprehension in normals and agrammatics. Cognition, 19, 133-166.

Friederici, A. D. (1995). The time course of syntactic activation during language processing: A model based on neuropsychological and neurophysiological data. Brain and Language, 50, 259-281.

Friederici, A. D., von Cramon, D. Y., \& Kotz, S. A. (1999). Language related brain potentials in patients with cortical and subcortical left hemisphere lesions. Brain, 122, 1033-1047.

Friederici, A. D., Hahne, A., \& von Cramon, D. Y. (1998). First-pass versus second-pass parsing processes in a Wernicke's and a Broca's aphasic: Electro-physiological evidence for a double dissociation. Brain and Language, 62, 311-341.

Friederici, A. D., Hahne, A., \& Mecklinger, A. (1996). The temporal structure of syntactic parsing: 
Early and late event-related brain potential effects elicited by syntactic anomalies. Journal of Experimental Psychology: Learning, Memory, and Cognition, 22, 1219-1248.

Friederici, A. D., \& Kilborn, K. (1989). Temporal constraints on language processing: Syntactic priming in Broca's aphasia. Journal of Cognitive Neuroscience, 1, 262-272.

Friederici, A. D., Kotz, S. A., Werheid, K., Hein, G., \& von Cramon, D. Y. (under revision). Syntactic comprehension in Parkinson's disease: Investigating early automatic and late integrational processes using ERPs.

Friederici, A. D., Pfeifer, E., \& Hahne, A. (1993). Event-related brain potentials during natural speech processing: Effects of semantic, morphological and syntactic violations. Cognitive Brain Research, 1, 183-192.

Grossman, M., Carvell, S., Gollomp, S., Stern, M. B., Vernon, R. N., \& Hurtig, H. I. (1991). Sentence comprehension and praxis deficits in Parkinson's disease. Neurology, 41, 1620-1626.

Grossman, M., Carvell, S., Stern, M. B., Gollomp, S., \& Hurtig, H. I. (1992). Sentence comprehension in Parkinson's disease: The role of attention and memory. Brain and Language, $42,347-384$

Grodzinsky, Y. (2000). The neurology of syntax: Language use without Broca's area. Behavioral and Brain Science, 23, 1-71.

Gunter, T. C., Friederici, A. D., \& Hahne, A. (1999). Brain responses during sentence reading: Visual input affects central processes. NeuroReport, 10, 3175-3178.

Gunter, T. C., Stowe, L. A., \& Mulder, G. (1997). When syntax meets semantics. Psychophysiology, $34,660-676$

Haarmann, H. J., \& Kolk, H. H. J. (1991). Syntactic priming in Broca's aphasia: Evidence for slow activation. Aphasiology, 5, 247-263.

Haarmann, H. J., \& Kolk, H. H. J. (1994). On-line sensitivity to subject-verb agreement violations in Broca's aphasics: The role of syntactic complexity and time. Brain and Language, 46, 493-516.

Hagoort, P. (1993). Impairments of lexical-semantic processing in aphasia: Evidence from the processing of lexical ambiguities. Brain and Language, 45, 189-232.

Hagoort, P., Brown, C., \& Groothusen, J. (1993). The syntactic positive shift as an ERP measure of syntactic processing. Language and Cognitive Processes, 8, 439-483.

Hagoort, P., Brown, C. M., \& Swaab, T. Y. (1996). Lexical-semantic event-related potential effects in patients with left hemisphere lesions and aphasia, and patients with right hemisphere lesions without aphasia. Brain, 119, 627-649.

Hahne, A., \& Friederici, A. D. (1999). Electrophysiological evidence for two steps in syntactic analysis: Early automatic and late controlled processes. Journal of Cognitive Neuroscience, 11, 194-205.

Kaan, E., Harris, A., Gibson, E., \& Holcomb, P. J. (2000). The P600 as an index of syntactic integration difficulty. Language and Cognitive Processes, 15, 159-201.

Kotz, S. A., Friederici, A. D., \& von Cramon, D. Y. (1999). Auditory word list priming in left and right temporal lobe lesion patients. Brain and Language, 69, 294-296.

Kutas, M., \& Federmeier, K. D. (2000). Electrophysiology reveals semantic memory use in language comprehension. Trends in Cognitive Sciences, 4(12), 463-470.

Kutas, M., \& Hillyard, S. A. (1980). Reading senseless sentences: Brain potentials reflect semantic incongruity. Science, 207, 203-205.

Kutas, M., \& Hillyard, S. A. (1983). Event-related potentials to grammatical errors and semantic anomalies. Memory and Cognition, 11, 539-550.

Kutas, M., \& Van Petten, C. (1988). Event-related potential studies of language. In P. K. Ackles, J. R. Jennings, \& M. G. H. Coles (Eds.), (Vol. 3). Advanced in psychophysiology, Greenwich: JAI Press.

Kutas, M., \& Van Petten, C. (1994). Psycholinguistics electrified: Event-related brain potential 
investigations. In M. A. Gernsbacher (Ed.), Handbook of psycholinguistics (pp. 83-143). San Diego: Academic Press.

Mecklinger, A., Schriefers, H., Steinhauer, K., \& Friederici, A. D. (1995). Processing relative clauses varying on syntactic and semantic dimensions: An analysis with event-related potentials. Memory and Cognition, 23, 477-494.

Milberg, W., Blumstein, S. E., \& Dworetzky, B. (1987). Processing lexical ambiguities. Brain and Language, 31, 138-150.

Milberg, W., Blumstein, S. E., Katz, D., Gershberg, F., \& Brown, T. (1995). Semantic facilitation in aphasia-Effects of time and expectancy. Journal of Cognitive Neuroscience, 7, 33-50.

Münte, T. F., Heinze, H. J., \& Mangun, G. R. (1993). Dissociation of brain activity related to syntactic and semantic aspects of language. Journal of Cognitive Neuroscience, 5, 335-344.

Münte, T. F., Heinze, H.-J., Matzke, M., Wieringa, B. M., \& Johannes, S. (1998). Brain potentials and syntactic violations revisited: No evidence for specificity of the syntactic positive shift. Neuropsychologia, 36, 217-226.

Neville, H. J., Nicol, J., Barss, A., Forster, K. I., \& Garrett, M. F. (1991). Syntactically based sentence processing classes: Evidence from event-related brain potentials. Journal of Cognitive Neuroscience, 3, 151-165.

Nobre, A. C., \& McCarthy, G. (1995). Language-related field potentials in the anterior-medial temporal lobe: II. Effects of word type and semantic priming. Journal of Neuroscience, 15, 1090-1098.

Osterhout, L., \& Holcomb, P. J. (1992). Event-related potentials and syntactic anomaly. Journal of Memory and Language, 31, 785-804.

Osterhout, L., \& Holcomb, P. J. (1993). Event-related potentials and syntactic anomaly: Evidence of anomaly detection during the perception of continuous speech. Language and Cognitive Processes, 8, 413-437.

Osterhout, L., Holcomb, P. J., \& Swinney, D. (1994). Brain potentials elicited by garden-path sentences: Evidence of the application of verb information during parsing. Journal of Experimental Psychology: Learning, Memory, and Cognition, 20, 786-803.

Osterhout, L., \& Mobley, L. A. (1995). Event-related brain potentials elicited by failure to agree. Journal of Memory and Language, 34, 739-773.

Penke, M., Weyerts, H., Gross, M., Zander, E., Münte, T. F., \& Clahsen, H. (1997). How the brain processes complex words: An ERP-study of German verb inflections. Cognitive Brain Research, $6,37-52$.

Pulvermüller, F. (1999). Words in the brain's language. Behavioral and Brain Sciences, 22, $253-336$.

Revonsuo, A., \& Laine, M. (1996). Semantic processing without conscious understanding in a global aphasic: Evidence from auditory event-related brain potentials. Cortex, 32, 29-48.

Rösler, F., Friederici, A. D., Pütz, P., \& Hahne, A. (1993). Event-related brain potentials while encountering semantic and syntactic constraint violations. Journal of Cognitive Neuroscience, 5 , $345-362$.

Swaab, T. A., Brown, C., \& Hagoort, P. (1997). Spoken sentence comprehension in aphasia: Eventrelated potentials evidence for a lexical integration deficit. Journal of Cognitive Neuroscience, 9 , 36-99.

Swaab, T. A., Brown, C., \& Hagoort, P. (1998). Understanding ambiguous words in sentence contexts: Electrophysiological evidence for delayed contextual selection in Broca's aphasia. Neuropsycholgia, 737-761.

Swinney, D., Zurif, E., \& Nicol, J. (1989). The effects of focal brain damage on sentence processing: An examination of the neurological organization of a mental module. Journal of Cognitive Neuroscience, 1, 25-37. 
Tyler, L. K., Ostrin, R. K., Cooke, M., \& Moss, H. E. (1995). Automatic access of lexical information in Broca's aphasics: Against the automaticity hypothesis. Brain and Language, 48, $131-162$.

Ullman, M. T., Corkin, S., Coppola, M., Hickok, G., Growdon, J. H., Koroshetz, W. J., \& Pinker, S. (1997). A neural dissociation within language: Evidence that the mental dictionary is part of declarative memory, and that grammatical rules are processed by the procedural system. Journal of Cognitive Neuroscience, 9, 266-276. 